\title{
Confidentiality-Preserving Query Execution of Fragmented Outsourced Data
}

\author{
Anis Bkakria ${ }^{1}$, Frédéric Cuppens ${ }^{1}$, Nora Cuppens-Boulahia ${ }^{1}$, \\ and José M. Fernandez ${ }^{2}$ \\ 1 Télécom Bretagne \\ 2 École Polytechnique de Montréal \\ \{anis.bkakria,frederic. cuppens, nora. cuppens\}@telecom-bretagne.eu, \\ jose.fernandez@polymtl.ca
}

\begin{abstract}
Ensuring confidentiality of outsourced data continues to be an area of active research in the field of privacy protection. Almost all existing privacy-preserving approaches to address this problem rely on heavyweight cryptographic techniques with a large computational overhead that makes inefficient on large databases. In this paper, we address this problem by improving on an existing approach based on a combination of fragmentation and encryption. We present a method for optimizing and executing queries over distributed fragments stored in different Cloud storage service providers. We then extend this approach by presenting a Private Information Retrieval (PIR) based query technique to enforce data confidentiality under a collaborative Cloud storage service providers model.
\end{abstract}

Keywords: Data confidentiality, Privacy-preserving, Data fragmentation, Data outsourcing.

\section{Introduction}

In the last few years, database outsourcing has become an important tool in IT management, as it offers several advantages to the client companies, especially for small ones with limited IT budget. In most models of database outsourcing, data storage and management (e.g. data backup and recovery) are completely operated by external service providers that take advantages of economies of scale to reduce the cost of maintaining computing infrastructure and data-rich applications in comparison with the cost of in-house data management.

Nonetheless, outsourcing gives rise to major security issues due to the usually sensitive nature of information saved in databases. Storing such sensitive information with external service providers requires them to be trusted and that they should protect data against external threats. Even if these service providers protect data adequately and process queries accurately, they may be curious about the stored data and may attempt to profit by disclosing this sensitive information to third parties. This raises the question of whether it is possible to protect confidentiality and privacy of the outsourced data. A recent study [10] 
by the Ponemon Institute indicates that since 2005 more than 250 million customer records containing sensitive information have been lost or stolen. The same study reports the average organizational cost of data leakage between 2005 and 2009 , to be about $\$ 6.6$ million. Hence, there seems to be a clear financial motivation to protect data privacy and confidentiality even when records are leaked.

Related Work. One approach to protect confidentiality and privacy of the outsourced data is based on encrypting all tuples in outsourced databases [8, 7]. With this approach, one crucial question is that of how to efficiently execute queries. Clearly, when using deterministic encryption techniques equality-match queries are simple to evaluate. However, range queries and aggregations become more difficult to perform as we must decrypt all records to evaluate this kind of queries, which makes query execution on the outsourced encrypted data much more difficult. Therefore, our main focus in this paper is about preserving both privacy and confidentiality of outsourced database while ensuring a secure way for querying outsourced data. One promising method to meet this requirement is to use data fragmentation. Basically, data fragmentation procedures are not particularly designed for preserving data security, they are aimed to improve data manipulation process, optimize storage, and facilitate data distribution. Nevertheless, in the last few years two significant alternatives have been proposed. The first one [9, 3] relies on data fragmentation alone to protect confidentiality. The distribution model used in this approach is composed mainly of two domains: a trusted local domain from which the data originates, and a honest but curious domain in which the data will be distributed. Because of its trustworthiness, the local domain is used to store fragments that contain highly sensitive data without the need to encrypt them. This is not so efficient in that it forces data owners to always protect and manage fragments containing highly sensitive data. A more promising alternative [4, 6] attempts to combine data fragmentation together with encryption. In this proposal, the main idea is to partition the data to be externalized across two or more independent service providers, and furthermore to encrypt all information which can not be secured by fragmentation (e.g. employees' bank account numbers of a company). While they do constitute an interesting way to ensure confidentiality of outsourced database, these approaches have the major limitation that it assumes that data to be outsourced is represented within a single relation schema (or table). Clearly, this assumption is too strong and seldom satisfied in the real environments, as generally, relational databases are normalized to minimize redundancy and dependency by dividing large tables into smaller (and less redundant) tables and defining relationships between them.

In this paper, we strive to protect the confidentiality and the privacy of sensitive outsourced database using both encryption and fragmentation, combining the best features of both approaches. Furthermore, we aim to overcome the previously mentioned limitations of [4, 6] by presenting an approach which is able to deal efficiently with multi-relation normalized databases. In a distributed 
environment, the problems encountered in one-relation 1 databases take on additional complexity when working with multi-relation normalized databases as it gives rise to new problems such as protecting the relatioships between relational schemas and defining a secure and efficient technique allowing authorized users to query these sensitive relationships. We will show how to protect data unlinkability of different fragments of the original database by protecting user query privacy using a practical Private Information Retrieval (PIR) technique. Unlinkability of two items of interest (e.g., records stored into different fragments) means that within the system, from an adversary point of view, these items of interest are no more and no less related. In our approach, a relation containing sensitive information will be fragmented into two or more fragments. Unlinkability of fragments means that despite the fact that an adversary has knowledge about the fragments of a relation, it remains unable to link records from different fragments.

The remainder of the paper is organized as follows. Section 2 illustrates through an example the problem and need for an approach like ours. In Section 3, we detail the threat model, security model, and assumptions. Section 4 describes our approach to enforce privacy and confidentiality of outsourced data. Section 5 presents the query optimization and execution model. In Section 6, we present a PIR-based technique to achieve query privacy and enforce data confidentiality under a collaborative Cloud storage service providers model. Finally, we conclude the paper in section 7 .

\section{Motivating Example}

Consider a relational hospital database $D$ with relations: Patient $(\underline{I d}$, Name,ZIP, Illness, Id_Doctor $\left.{ }^{*}\right)$ and $\operatorname{Doctor}(\underline{\mathrm{Id}}$, Name, Specialty) where Id_Doctor is a foreign key referencing the column $I d$ of the relation Doctor. Let us assume that the hospital is going to outsource the database to a third party. Sensitive information contained in $D$ should be protected. If we look carefully, we can consider that the list of patients and their attributes (Id, Name, Zip) are not sensitive, and also that the list of illnesses could be made public. Nevertheless, the relationship between these two lists (list of patients and list of illnesses) should be protected. Therefore if we can find a way (e.g. vertical fragmentation [1]]) to break relationships between patients and their respective illnesses, there is no need to encrypt all records of the Patient relation. On the other hand, the relationship between a patient and his doctor should be protected. Since the list of doctors itself is not sensitive, the simplest way to protect the relationship between the two relations Patient and Doctor consists in encrypting the foreign key $I d_{-}$Doctor. Actually, we can either encrypt the foreign key $I d \_D o c t o r$ of the relation Patient or the primary key $I d$ of the relation Doctor, because in the two cases, relationship between relations Patient and Doctor will be protected. However, encrypting the foreign key seems to be more beneficial as a foreign key references only one relation (only the relationship between the two relations is

\footnotetext{
${ }^{1}$ Databases composed from a single relation shema.
} 
protected) while a primary key can be referenced by many relations. Therefore, if we encrypt the primary key, we will protect all relationships between the relation containing the primary key and other relations referencing the encrypted primary key. Thus, when the security requirement specifies that only the a relationship between data is sensitive, our apporach is more appropriate than the one based on full encryption.

\section{Threat Model and Security Assumptions}

Our approach is based on a typical client-server architecture, where servers are managed by different service providers. These service providers are considered "honest-but-curious", in agreement with most related work [3, 4, 7, 9]. Service providers are assumed to be "honest" in that they do not manipulate outsourced data in order to respond incorrectly to user queries. In other words, we suppose that responses to user queries received from these service providers are always accurate. In the first part of this paper, we will assume that service providers are "curious" in that they will try to infer and analyze outsourced data, and will also actively monitor all received user queries and try to derive as much information as possible from these queries. In the second part of the paper, we further assume that service providers can collude and cooperate together to link outsourced data. The client part of this architecture is assumed to be trustworthy and all interactions between the user and the client are secured. Protecting the client part against external attacks is beyond the scope of this article.

\section{Confidentiality Using Fragmentation and Encryption}

Our approach extends in several ways the vertical fragmentation-based approach described in [4, 6]. This approach considers that all data is stored in a single relation, while in our approach data can be stored in several relations, which is the case in typical database environments. In our approach, we consider that databases to be externalized are normalized so that two relations can be only associated together through a primary key/foreign key relationship. For this purpose, we introduce a new type of confidentiality constraint for fragmentation, the inter-table fragmentation constraint. The aim of this new fragmentation constraint is to protect the relationship between relations. This section first presents the different kinds of confidentiality constraints used to achieve our goals of protecting the confidentiality by encryption and fragmentation, and second formalises the concept of fragmentation in our approach which extends ideas presented in [4] 6 .

Definition 1 (Confidentiality Constraint). Consider that data to be secured are represented with a relational database $D$, which is composed of a list of relational schemas $R=\left(R_{1}, \ldots, R_{n}\right)$, with each of these relational schemas $R_{i}$ containing a list of attributes $A_{R_{i}}=\left(a_{1, i}, a_{2, i}, \ldots\right)$. A confidentiality constraint over $D$ can be one of the following: 
Singleton Constraint $(S C)$. It is represented as a singleton set $S C_{R_{i}}=\left\{a_{j, i}\right\}$ over the relation $R_{i}$. This kind of confidentiality constraint means that the attribute $a_{j, i}$ of the relational schema $R_{i}$ is sensitive and must be protected, typically by applying encryption.

Association Constraint $(A C)$. This kind of confidentiality constraint is represented as a subset of attributes $A C_{R_{i}}=\left\{a_{1, i}, \ldots, a_{j, i}\right\}$ over the relational schema $R_{i}$. Semantically, it means that the relationship between attributes of the subset $A C_{R_{i}}$ is sensitive and must be protected.

Inter-table Constraint $(I C)$. It is represented as a couple of relational schemas $I C=\left\{R_{i}, R_{j}\right\}$ of the relational database $D$. Relations $R_{i}$ and $R_{j}$ should be associated through a primary key/foreign key relationship. The use of this kind of confidentiality constraint ensures protection of the primary key/foreign key relationship between the two relational schemas concerned with the inter-table constraint $I C$.

Note that protecting the relationship between two tables relies on protecting the primary key/foreign key relationship and storing involved relations separately. The association constraint can also be addressed through encryption (encrypt at least one of attributes involved in the constraint), but clearly this will increase the number of encrypted attributes and make interrogation of the database more complicated. A more adapted way to resolve this kind of confidentiality constraint was proposed in [4], which is based on splitting involved attributes in a manner that their relationships cannot be reconstructed.

In the case of an inter-table confidentiality constraint, protecting the foreign key using encryption is the simplest way to secure the relationship between the two relational schemas. However encrypting only the foreign key is not enough to keep the relationship between relational schemas secure, as service provider may be able to link records in two relational schemas by observing and analyzing user queries over these relational schemas. To overcome this problem, the two relational schemas in question should be split into different fragments, and each of these fragments should be distributed to a different Cloud storage provider. An interesting approach for modeling constraints and resolving the data fragmentation problem was proposed in [6], that efficiently computes data fragments that satisfy the confidentiality constraints. It is based on Boolean formulas and Ordered Binary Decision Diagrams (OBDD) and uses only attribute-based confidentiality constraint (Singleton Constraints and Association Constraints). However, it cannot deal as-is with Inter-table Constraints. In order to use this approach, we define a way to reformulate Inter-table Constraint as a set of Singleton Constraints and Association Constraints. We explain this transformation in the definition and theorem below.

Definition 2 (Inter-table Constraint transformation). Consider a relational database with two relations $R_{1}\left(\underline{a_{1}}, \ldots, a_{n}\right)$ and $R_{2}\left(\underline{b_{1}}, \ldots, b_{m} *\right)$. Let us assume that $R_{1}$ and $R_{2}$ are related through a foreign key/primary key relationship in which the foreign key $b_{m}$ of the relation $R_{2}$ references the primary key $a_{1}$ of relation $R_{1}$. We assume that $R_{1}$ and $R_{2}$ contain respectively $p$ and $q$ records, 
with $p>1$ and $q>1$. An Inter-table Constraint $c=\left\{R_{1}, R_{2}\right\}$ over relations $R_{1}$ and $R_{2}$ states that the relationship between these two relations must be protected by encrypting the foreign key $b_{m}$ and by storing $R_{1}$ and $R_{2}$ in two different fragments. Therefore, the constraint $c$ can be written as follows:

i A singleton constraint $S C=\left\{b_{m}\right\}$ to state that the value of $b_{m}$ should be protected.

ii A list of $(m \times n)$ association constraints $A C=\left\{\left(a_{i}, b_{j}\right) \mid i \in[1, n], j \in[1, m]\right\}$.

We propose the notion of a correct transformation of Inter-table constraints. A transformation of an Inter-table constraint $c$ to a set of confidentiality constraints $C$ is correct if the satisfaction of $C$ enforce the protection of the unlinkability between records of the two relations involved in $c$. The following Theorem formalizes this concept.

Theorem 1 (Transformation correctness). Given a relational database $\mathcal{D}$ composed from two relational schemas $R_{1}\left(\underline{a_{1}}, \ldots, a_{n}\right)$ and $R_{2}\left(\underline{b_{1}}, \ldots, b_{m} *\right)$ related through relationship between the foreign key $b_{m}$ of $R_{2}$ and the primary key $a_{1}$ of $R_{1}$. Let $c=\left\{R_{1}, R_{2}\right\}$ be an Inter-table constraint, the set of constraints $C$ be the result of the transformation of $c$, and $\mathcal{F}=\left\{F_{1}, \ldots F_{q}\right\}$ be a fragmentation of $\mathcal{D}$ that satisfies $C$. The Inter-table constraint $c$ is correctly transformed into a set of constraints $C$ if all the following conditions hold :

1. $b_{m}$ does not appear in clear in any fragment of $\mathcal{F}$.

2. $\forall A C_{i, j}=\left\{a_{i}, b_{j}\right\} \in C, i \in[1, n], j \in[1, m], \quad$ if $a_{i} \in F_{k}$ and $b_{j} \in$ $F_{l}$ then $k \neq l$

The main advantage of the Inter-table constraint is that it allows treatment of multi-table relational databases. In addition, it gives a simple way to formulate confidentiality constraints between relations. As we have seen in Item (i) of Definition 2, the attribute $b_{m}$ (foreign key of the relation $R_{2}$ ) should be encrypted. However, to be able to query data and construct relationship between relations, the chosen encryption algorithm must be deterministic [1] in order to preserve uniqueness and allow the construction of relationship between relations (e.g. through JOIN queries). As is known, in normalized multi-relation databases, three types of relationship between relations exist: (1) one-to-one, (2) one-to-many and (3) many-to-many relationships. Inter-table constraints over relations associated using (1) or (2) can be simply transformed as shown in Definition 2, while others associated using (3) need a pre-transformation step before applying the transformation of Definition 2, as they are normally linked through a third relation known as a linking table. The pre-transformation steps is described in the example below.

\section{Definition 3. Fragmentation [5]}

Let us consider a relational database $D$ with relations $R_{1}, \ldots, R_{n}$ and $A$ the list of all attributes contained in different relations. Given $A_{f}$ the list of attributes to be fragmented, the result of the fragmentation is a list of fragments $F=$ $\left\{F_{1}, \ldots, F_{m}\right\}$ where each of these fragments satisfies: 


$$
\begin{aligned}
& \forall F_{i} \in F, i \in[1 . . m], F_{i} \subseteq A_{f} . \\
& \forall a \in A_{f}, \exists F_{i} \in F: a \in F_{i} . \\
& \forall F_{i}, F_{j} \in F, i \neq j: F_{i} \cap F_{j}=\emptyset .
\end{aligned}
$$

Note that the list of attributes to be fragmented $A_{f}$ contains all attributes in $A$, except those concerned with Singleton Constraints (attributes to be encrypted). Condition (i) guarantees that only attributes in $A_{f}$ are concerned by the fragmentation, condition (ii) ensures that any attribute in $A_{f}$ appears in clear at least in one fragment and condition (iii) guarantees unlinkability between different fragments.

Logically, to be able to get information about the original database, we should be able to reconstruct original database from fragments. So after defining the fragmentation process, we shall define a mechanism to combine fragmentation and encryption. More clearly, a mechanism to integrate attributes involved in the Singleton Constraints (attributes to be encrypted) in the suitable fragment. These encrypted attributes allow only authorized users (users who know the encryption key) to construct the sensitive relationships. Based on the definition of Physicalfragment proposed in [4], we define our mechanism called Securefragment to combine fragmentation and encryption.

Definition 4. Secure Fragment: Let $D$ be a relational database with a list of relations $R=\left\{R_{1}\left(a_{1,1}, \ldots, a_{j, 1}\right), \ldots, R_{n}\left(a_{1, n}, \ldots, a_{k, n}\right)\right\}, F=\left\{F_{1}, \ldots, F_{m}\right\}$ a fragmentation of $\mathrm{D}$ and $A_{f}$ be the list of fragmented attributes. Each fragment $F_{i} \in F$ is composed of a subset of attributes $A_{i} \subseteq A_{f}$. Each $A_{i}$ is composed of a subset of attributes of one or more relations $R_{j} \in R$. We denote by $R_{F_{i}}$ the list of relations in $R$ which a subset of their attributes belongs to the fragment $F_{i} \in F$. The secure fragment of $F_{i}$ is represented by a set of relations schema $R_{F_{i}}^{e}$ in which each relation is represented as follows $R_{j}^{e}\left(\underline{\text { salt }}\right.$, enc $\left., a_{1}, \ldots, a_{k}\right)$ where $\left\{a_{1}, \ldots, a_{k}\right\} \subset A_{i} \cap R_{j}$ and $e n c$ is the encryption of all attributes of $R_{j}$ that do not belong to $\left\{a_{1}, \ldots, a_{k}\right\}$ (all attributes of $R_{j}$ involved in a singleton constraint except those concerned by a singleton constraint over the foreign key), combined before encryption in a binary XOR with the salt. All foreign key attributes which are involved in singleton constraints are encrypted using a deterministic encryption algorithm (e.g., AES) to ensure their indistinguishability. The Algorithm 1 shows the construction of secure fragments. The main reason for reporting all original attributes (except foreign keys involved in the Singleton constraints) in an encrypted form for each relation in a fragment, is to guarantee that a query $Q$ over the original relation $R_{j}$ can be executed by querying a single fragment (which contains $R_{j}^{e}$ ) while preserving confidentiality of sensitive relationships, so we do not need to reconstruct the original relation $R_{j}$ to perform the query $Q$. Furthermore, encrypting foreign keys ensure the protection of sensitive relationships between relations involved into Inter-table constraints. However, using deterministic encryption algorithm has two issues. First, a major advantage is to enforce indistinguishability of records which allows only authorized users who know the encryption key to execute queries associating these relations. Second, a minor drawback is that it allows an adversary to infer information about repeatedly occurring values of the encrypted foreign keys, but this information 
does not allow the adversary to break the unlinkability between relations. The attribute salt which is used as a primary key of different relations in the secure fragments protects encrypted data against frequential attacks. In addition, there is no need to secure the salt attribute because knowledge of the value of this attribute will not give any advantage when attacking encrypted data.

Example 4.2. Assume that we have a relational database $D$ of a medical insurance company that contains two relations Patient and Doctor represented respectively in Table1 and Table 2. The insurance company has defined the a set of confidentiality constraints $C C=\left\{C_{1}=\{S S N\}, C_{2}=\{\right.$ Name_pat, Illness $\}$, $C_{3}=\{$ Patient, Doctor $\}$. As shown before, the first step in the fragmentation process consists in transforming Inter-table constraint $\left(C_{3}\right)$. Relations Patient and Doctor are linked through the foreign key $I d_{-} d o c$ in the relation Patient, therefore $C_{3}$ will be replaced by $C_{4}=\left\{I d_{\_} d o c\right\}$ and all possible Association constraints composed of an attribute of the relation Doctor and an attribute of the relation Patient (Guarantee that the relation Patient will not be in the same fragment as the relation Doctor). In our example, attributes $S S N$ and $I d \_d o c$ of the relation Patient are involved in singleton constraints $C_{1}$ and $C_{4}$ respectively. So they will not be concerned by the fragmentation. As a result $C_{3}$ will be replaced by :

$$
\begin{aligned}
& \text { - } C_{4}=\left\{I d_{-} d o c\right\} \quad \bullet C_{8}=\{\text { Dob,Name_doc }\} \\
& \text { - } C_{5}=\{\text { Name_pat,Id_doctor }\} \cdot C_{9}=\{\text { Illness, Id_doctor }\} \\
& \text { - } C_{6}=\{\text { Name_pat,Name_doc }\} \bullet C_{10}=\{\text { Illness, Name_doc }\} \\
& \text { - } C_{7}=\{\text { Dob, Id_doctor }\}
\end{aligned}
$$

Table 1. Patient relation

\begin{tabular}{|l|l|l|l|l|}
\hline SSN & Name_pat & Dob & Illness & Id_doc \\
\hline \hline 865746129 & A. Barrett & $20-08-1976$ & Illness & doc_3 \\
591674603 & C. Beat & $18-01-1981$ & Illness $_{2}$ & doc_3 \\
880951264 & N. Baines & 14-09-1986 & Illness $_{1}$ & doc_2 $^{\prime}$ \\
357951648 & S. Brandt & 18-01-1981 & Illness $_{3}$ & doc_1 \\
\hline \hline
\end{tabular}

Table 2. Doctor relation

\begin{tabular}{|l|l|}
\hline Id_doctor & Name_doc \\
\hline doc_1 & C. Amalia \\
doc_2 & D. Annli \\
doc_3 & P. Amadeus \\
\hline \hline
\end{tabular}

A possible fragmentation of $D$ that satisfies all confidentiality constraints is the set of fragments $\left\{F_{1}, F_{2}, F_{3}\right\}$ with: $F_{1}=\left\{\right.$ Patient $($ Name_pat, Dob) $\}, F_{2}=$ $\{$ Patient(Illness) $\}$ and $F_{3}=\left\{\right.$ Doctor $\left(I d_{-}\right.$doctor, Name_doc $\left.)\right\}$. Next step is the Securefragmentation transformation (Definition 3 ). We assume that encryption of the protected attributes uses the deterministic encryption algorithm $E$ with the encryption key $K$. The result of applying the SecureFragmentation over different fragments is represented as follows.

- $F_{1}$ : Patient (salt, enc, Name_pat, Dob, $\left.E_{k}\left(I d \_d o c\right)\right)$ with

enc $=E_{K}(\langle S S N$, Illness $\rangle \bigoplus$ salt $)$

- $F_{2}$ : Patient (salt, enc, Illness, $\left.E_{k}\left(I d \_d o c\right)\right)$ with

enc $=E_{K}\left(\left\langle S S N, N a m e_{-} p a t, D o b\right\rangle \bigoplus\right.$ salt $)$

- $F_{3}$ : Doctor(Id_doctor, Name_doc) 
Note that $F_{3}$ has not been changed because there is no singleton constraints over the Doctor attributes. Lastly data fragments $F_{1}, F_{2}$ and $F_{3}$ are distributed to different Cloud storage providers.

\section{$5 \quad$ Query Execution Model}

Before discussing techniques for processing query over distributed fragments, we will first present the architecture of our proposed approach. It includes three principal entities : (1) a User which attempts to execute queries over the original database, (2) a Client which rewrites user queries by splitting them to create an optimized distributed Query Execution Plan QEP; QEP is a set of sub-queries and other operations (e.g., decryption, join...), it is created by the Query Transformative based on the MetaData which contains information (relations, clear attributes, encrypted attributes, selectivity of attributes) about data distribution in different fragments. Furthermore, the Query Executor executes each of these sub-queries over the appropriate fragments and sends back the results to the client. (3) Server represented by different Cloud storage providers in which data fragments are distributed.

Query Transformation and Optimization. In our querying model, query transformation is performed by the Query Transformative $(Q T)$ entity on the client side. When receiving a user query, the query is analyzed syntactically and semantically so that incorrect queries are rejected as earlier as possible. Next, based on the Metadata stored on the client side, the $Q T$ will attempt to find a fragment on which the user query can be executed, i.e. a fragment in which $Q T$ can find all attributes and relations involved in the user query. If such a fragment does not exist, $Q T$ will decompose the user query into queries expressed in relational algebra, find out which fragments are involved in the query, and finally transform the user query into a set of fragments queries. Using this set of fragment queries and other operations such as encryption, decryption, join and aggregation, the $Q T$ creates a QEP and sends it to the Query Executor. A query can have more that one QEP. Logically, each QEP may have a different execution cost. Thus, the $Q T$ should have the capability to pick out the best QEP in terms of execution cost. This capability is explained later in the Query Optimization section.

For multi-fragment query 2, $Q T$ will use local join operations as it should combine results of execution of subqueries over fragments. There are two different ways to perform local join operation : (1) Execute all sub-queries in a parallel manner, then join the result on the client side. (2) Execute sub-queries in a sequential manner to have the ability to perform semi-joins using the result of previous sub-queries. While (1) can be cheaper than (2) in terms of sub-query execution, it is much more costly in the join operation because in (1), sub-queries results might contain a lot of records that will not be part of the final results.

\footnotetext{
2 i.e. a query that cannot be executed over only one fragment.
} 
In addition to traditional query optimization methods such as selecting conditions as earlier as possible, the $Q T$ attempts to minimize the execution cost of the created QEP by applying the selection condition with the most selective attribute, i.e the selection condition which is satisfied by the smallest number of tuples. To give this ability to the $Q T$, we assign a selectivity ${ }^{3}$ to each attribute contained in the original database to the Metadata stored in the Client. We apply the optimization method to the example below.

\section{Preserving Data Unlinkability}

Ensuring data confidentiality is achieved by preserving unlinkability between different data fragments and by encrypting all sensitive information that cannot be protected using only fragmentation. However, we have seen in the previous section that evaluation of some queries may use semi join in order to join data from different fragments. This will not be a concern in the case of non-colluding Cloud storage providers, but it becomes a serious concern when Cloud Storage Providers (CSP) can collude. In this section, we present our solution to overcome this privacy concern when we assume that CSP can collude to link data stored in different fragments.

To overcome this problem, the Client should have the ability to execute semi join queries and retrieve data from a fragment without the CSP (which stores the fragment) learning any information about the semi join condition values. To meet this requirement, we will use a Private Information Retrieval keyword-based technique. PIR keyword-based was presented in [2] to retrieve data with PIR using keywords search over many data structures such as binary trees and perfect hashing. Later, [12] investigated the use of SQL for PIR, based on the use of $\mathrm{B}+$ tree. In the next part of this paper, we will explain how we can use technique presented in [12] to ensure our semi join queries privacy requirement.

Theorem 2. Let $\mathcal{D}$ be a multi-relation normalized database, $\mathcal{F}=\left\{F_{1}, F_{2}\right\}$ be a fragmentation of $\mathcal{D}$, and $Q$ be a multi-fragment query that joins records from both fragments $F_{1}$ and $F_{2}$. Consider that SCPs in which the fragments $F_{1}$ and $F_{2}$ are stored can collude to link data stored in these fragments, and that $Q$ is evaluated using semi join operations. Sensitive relationships between $F_{1}$ records and $F_{2}$ records remain protected if and only if the privacy of the semi join subqueries is guaranteed.

PIR System Design. In the Client of our architecture, we give to Query Executor the ability to communicate with different Cloud storage providers through the PIR keyword-based protocol. In the Server, we add on each CSP a PIR Server as a front-end entity to answer Query Executor's PIR queries. An adversary (a Cloud storage provider administrator) who can observe Query Executor's PIR-encoded queries is unable to find out the clear content of the queries. Enforcing integrity on the PIR server side is straightforward since we

\footnotetext{
${ }^{3}$ Provides an approximation of the number of tuples that satisfies a predicate.
} 
assume that PIR servers will not attempt to wrongly answer Query Executor's PIR queries.

Keyword Index Structures. The main purpose for using PIR keyword-based is to ensure the privacy of semi join queries. In our approach, this kind of queries is mainly executed over primary or foreign key attributes. Therefore each PIR Server will create a B+ Tree over each indexed attribute (Primary or foreign key). The advantage of such an index structure is that data appears only in the leaves while other nodes are used to control the search. On the leaves of $\mathrm{B}+$ tree and for each key in the tree, we store the tuple corresponding to the key as the data part linked to the key. We consider the act of retrieving a node's data as a PIR operation over all nodes in the B+ tree. In all existing PIR schemes, a common assumption is that the client should know the address of the block or the item to be retrieved. To satisfy this assumption in our approach, the PIR server after creating the index structure, sends an index helper containing the $\mathrm{B}+$ tree's root node to the client.

Semi-Join PIR Keyword-based Query. Using the PIR keyword-based query requires a setup phase in which the Query Executor and the PIR server exchange information. This setup phase is divided into two steps:

1. The Query Executor sends the Relation schema name and the attribute name over which the semi join query is to be performed to the corresponding PIR server.

2. When receiving the Relation schema name and the attribute name, the PIR server selects the corresponding $\mathrm{B}+$ tree and sends its root node to the Query Executor.

After receiving the root node sent by the PIR server, the Query Executor will compare the list of keys contained in the root node with values used in the condition of the Semi join query in order to find out the indexes of the next nodes to be retrieved. The Query Executor will subsequently perform PIR queries over chosen indexes to retrieve corresponding nodes. Once all items have been retrieved, The Query Executor combines them to build the result of the original Semi join query. Refer to Appendix D for a description of the PIR keyword-based protocol algorithms used in the Client and the Server parts.

\section{Conclusion}

Existing approaches based on fragmentation and encryption have focused on single-relation schema database which is a strong and rarely encountered assumption in real environment. In this paper, we have presented a new approach based on fragmentation, encryption and query privacy techniques which ensures confidentiality of outsourced multi-relation databases. We have presented also a way to optimize and execute queries over distributed data fragments.

Our future work will include the implementation of our approach. It will also include enhanced query optimization and execution techniques to overcome some limitations of our approach, such as processing nested queries. 


\section{References}

1. Bellare, M., Fischlin, M., Ristenpart, T.: Deterministic encryption: Definitional equivalences and constructions without random oracles (2008)

2. Benny Chor, N.G., Naor, M.: Private information retrieval by keywords. Cryptology ePrint Archive, Report 1998/003 (1998)

3. Biskup, J., Preuß, M., Wiese, L.: On the Inference-Proofness of Database Fragmentation Satisfying Confidentiality Constraints. In: Lai, X., Zhou, J., Li, H. (eds.) ISC 2011. LNCS, vol. 7001, pp. 246-261. Springer, Heidelberg (2011)

4. Ciriani, V., De Capitani di Vimercati, S., Foresti, S., Jajodia, S., Paraboschi, S., Samarati, P.: Fragmentation and Encryption to Enforce Privacy in Data Storage. In: Biskup, J., López, J. (eds.) ESORICS 2007. LNCS, vol. 4734, pp. 171-186. Springer, Heidelberg (2007)

5. Ciriani, V., De Capitani di Vimercati, S., Foresti, S., Jajodia, S., Paraboschi, S., Samarati, P.: Fragmentation design for efficient query execution over sensitive distributed databases. In: ICDCS, pp. 32-39. IEEE Computer Society (2009)

6. Ciriani, V., De Capitani di Vimercati, S., Foresti, S., Livraga, G., Samarati, P.: Enforcing Confidentiality and Data Visibility Constraints: An OBDD Approach. In: Li, Y. (ed.) DBSec. LNCS, vol. 6818, pp. 44-59. Springer, Heidelberg (2011)

7. Hacigümüs, H., Iyer, B.R., Li, C., Mehrotra, S.: Executing sql over encrypted data in the database-service-provider model. In: SIGMOD Conference, pp. 216-227. ACM (2002)

8. Hacigümüs, H., Mehrotra, S., Iyer, B.R.: Providing database as a service. In: ICDE, pp. 29-38. IEEE Computer Society (2002)

9. Hudic, A., Islam, S., Kieseberg, P., Weippl, E.R.: Data confidentiality using fragmentation in cloud computing. International Journal of Communication Networks and Distributed Systems, IJCNDS (2012)

10. Ponemon Institute. Fourth annual us cost of data breach study (January 2009)

11. Ceri, S., Wiederhold, G., Navathe, S.B., Dou, J.: Vertical partitioning of algorithms for database design. ACM Trans. Database Syst. 9(4), 680-710. 98, 99, 102, 109, 125 (1984)

12. Olumofin, F., Goldberg, I.: Privacy-Preserving Queries over Relational Databases. In: Atallah, M.J., Hopper, N.J. (eds.) PETS 2010. LNCS, vol. 6205, pp. 75-92. Springer, Heidelberg (2010)

\section{A Secure Fragmentation Algorithm}

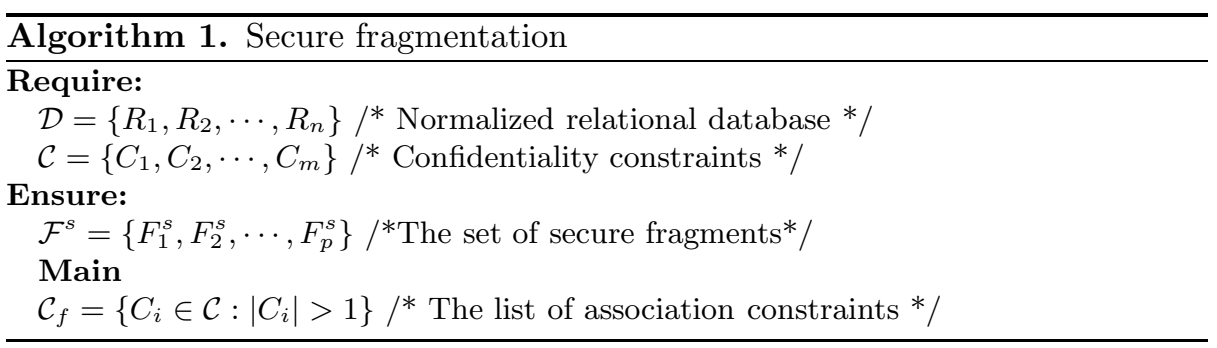




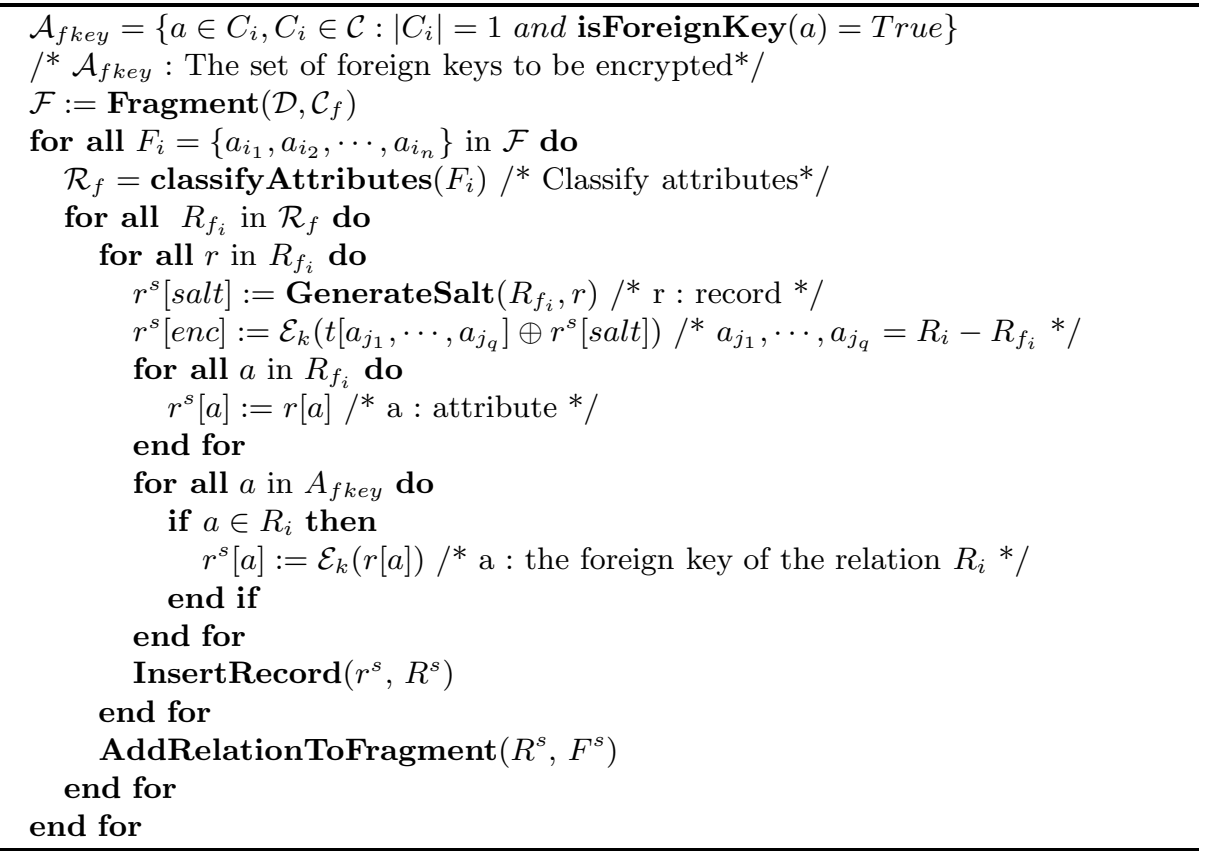

\section{B Proof of Theorem 1}

Proof. According to Item (ii) of Definition 2, the Inter-table constraint will be replaced by all possible associations constraint composed from an attribute of relation $R_{1}$ and another from relation $R_{2}$. Due to the fact that an association constraint between two attributes means that the relationship between these attributes will be protected using fragmentation (each attribute will be stored in different fragments), Item ( $i i)$ guarantees that relations $R_{1}$ and $R_{2}$ will be stored in different fragments which hold condition (2).

Item $(i)$ of Definition 2 creates a singleton constraint over the foreign key $b_{m}$ of the relation $R_{2}$. Thus $b_{m}$ will be considered as a sensitive attribute and will be protected using encryption, which means that the foreign key $b_{m}$ will not appear in clear in any fragment. As a result, if an adversary succeeds in having access to the fragments in which $R_{1}$ and $R_{2}$ have been stored, she is unable to link data stored in these relations.

\section{Proof of Theorem 2}

Proof. To prove the Theorem 2, we will use the following two sketches. The fist sketch proves that without ensuring semi join sub-queries privacy, collaborative CSPs can, in some cases, break data unlinkability, while the second sketch proves that, under a collaborative Cloud storage service providers model, 
protecting data unlinkability can only be guaranteed with the protection of the privacy of the semi join sub-queries.

SKETCH Without Using the PIR Keyword-based Protocol. Suppose that the $C$ ient wants to execute a query which joins records from two fragments $F_{1}$ and $F_{2}$. Let us consider that the sub-query $Q_{1}$ executed over the fragment $F_{1}$ has returned $n$ tuples. And the semi-join query $Q_{2}$ executed over $F_{2}$ has returned $m$ tuples. Therefore, if CSPs that store $F_{1}$ and $F_{2}$ collude together to link tuples from $Q_{1}$ and $Q_{2}$ results, the probability to guess correctly the relationship between tuples is:

$$
\operatorname{PROB}\left[\operatorname{Result}\left(Q_{1}\right) \leftrightarrow \operatorname{Result}\left(Q_{2}\right)\right]=\frac{1}{m \times n}
$$

Clearly, if $m$ and $n$ are small, CSPs will have a great chance to break data unlinkability.

SKETCH Using the PIR Keyword-based Protocol. Let us consider that the Client attempts to perform a query which joins records from two fragments $F_{1}$ and $F_{2}$. According to our defined PIR keyword-based protocol, the Client will execute $Q_{1}$ over the fragment $F_{1}$ without using the keyword-based protocol. Next, the Client will send the table name $T$ and the attribute name $a$ on which the semi-join will be performed, the Server replies with the root node of the corresponding $\mathrm{B}+$ tree. It is clear from the previous step that the CSP which stores $F_{2}$ can only know the attribute name and the table name on which the semi-join will be performed. After receiving the root node, the Client will use the PIR protocol to retrieve internal corresponding nodes until the leaves of the $\mathrm{B}+$ tree are reached. The PIR protocol will ensure that the server will not know which nodes were retrieved by the Client. Moreover, all tuples are stored in the leaf level of the $\mathrm{B}+$ tree. Therefore, in order to retrieve each record, the Client shall execute the same number of PIR queries. Rightfully, the only revealed information when using the PIR keyword-based protocol is the table name and the attribute name on which the semi-join has been performed. Therefore, if CSPs storing $F_{1}$ and $F_{2}$ collude together to break data unlinkability, they will be able only to infer that the relation $T_{1}$ in $F_{1}$ over which $Q_{1}$ has been executed is linked to the relation $T$ through the attribute $a$. Due to the fact that the foreign key in $T_{1}$ referencing the attribute a in $T$ is encrypted, linking records is not possible. 


\section{SemiJoin PIR Keywordbased Query Algorithms}
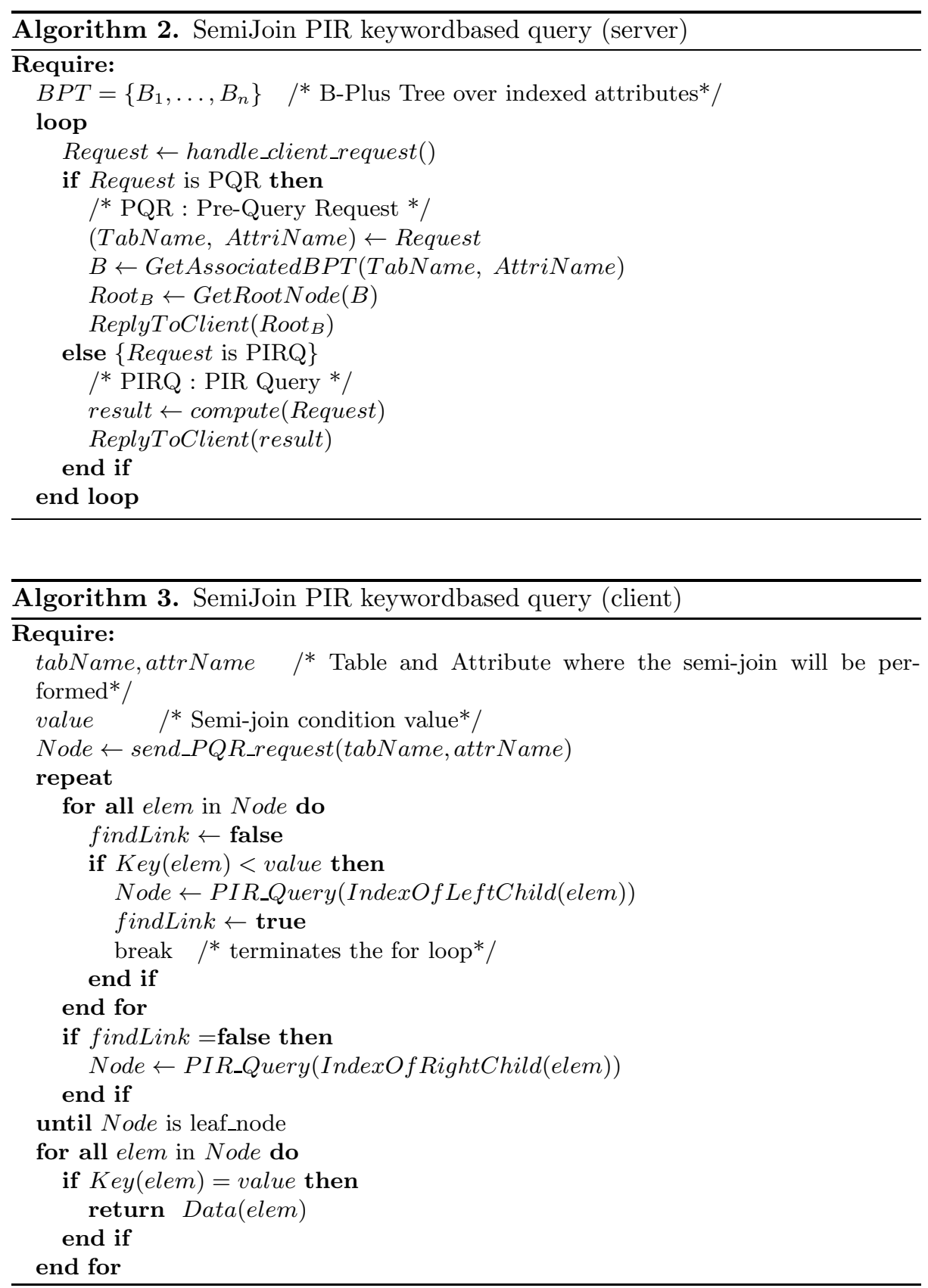